\title{
Recombinant CCL17 Enhances Hematoma Resolution and Activation of CCR4/ERK/Nrf2/CD163 Signaling Pathway After Intracerebral Hemorrhage in Mice
}

\author{
Shuixiang Deng ${ }^{1,2} \cdot$ Prativa Sherchan ${ }^{2} \cdot$ Peng Jin ${ }^{1,2} \cdot$ Lei Huang $^{3} \cdot$ Zachary Travis $^{2}$. \\ John H. Zhang ${ }^{2,3,4} \cdot$ Ye Gong ${ }^{1}$ • Jiping Tang ${ }^{2,5}$ (B)
}

Published online: 11 August 2020

(C) The American Society for Experimental NeuroTherapeutics, Inc. 2020

\begin{abstract}
Hematoma is a crucial factor leading to poor prognosis after intracerebral hemorrhage (ICH). Promoting microglial phagocytosis to enhance hematoma resolution may be an important therapeutic target for recovery after ICH. C-C chemokine receptor 4 (CCR4) is important for regulating immune balance in the central nervous system. However, whether CCR4 activation can attenuate hematoma after ICH remains unknown. We aimed to evaluate whether CCL17 (a specific ligand of CCR4) treatment can promote hematoma resolution through CCR4/ERK/Nrf2/CD163 pathway after ICH. A total of 261 adult male CD1 mice were used. Mice were subjected to intrastriatal injection of autologous blood to induce ICH and randomly assigned to receive recombinant CCL17 (rCCL17) or vehicle which was administered intranasally at $1 \mathrm{~h}$ after ICH. To elucidate the underlying mechanism, C021, a selective inhibitor of CCR4 and ML385 and a selective inhibitor of Nrf2 were administered $1 \mathrm{~h}$ prior to ICH induction. Clustered regularly interspaced short palindromic repeats (CRISPR) knockout for CD163 was administered by intracerebroventricular injection at $48 \mathrm{~h}$ before ICH. Brain edema, short- and long-term neurobehavior evaluation, hematoma volume, hemoglobin content, western blot, and immunofluorescence staining were performed. Endogenous CCL17, CCR4, and CD163 expression increased and peaked at $72 \mathrm{~h}$ after ICH. CCR 4 was expressed by microglia. CCR4 activation with rCCL17 significantly improved neurobehavioral scores and reduced hematoma volume and brain edema compared with vehicle. Moreover, rCCL17 treatment significantly promoted phosphorylation of ERK1/2, increased the expression Nrf2, and upregulated CD163 expression after ICH. The protective effects of rCCL17 were abolished by administration of C021, ML385, and CD163 CRISPR knockout. This study demonstrated that CCR4 activation with rCCL17 promoted hematoma resolution by increasing CD163 expression and CCR4/ERK/Nrf2 pathway activation after ICH, thereby reducing brain edema and improving neurological function. Overall, our study suggests that CCR4 activation may be a potential therapeutic strategy to attenuate hematoma in early brain injury after ICH.
\end{abstract}

Key Words Intracerebral hemorrhage $\cdot$ recombinant CCL17 $\cdot \mathrm{C}-\mathrm{C}$ chemokine receptor $4 \cdot$ scavenger receptor CD163 $\cdot$ hematoma resolution

Ye Gong

drgongyeicu@163.com

Jiping Tang

jtang@1lu.edu

1 Department of Critical Care Medicine, HuaShan Hospital, Fudan University, 12 Middle WuLuMuQi, Shanghai 200040, China

2 Department of Physiology and Pharmacology, Center for Neuroscience Research, Loma Linda University School of Medicine, Loma Linda, California 92350, USA
3 Department of Neurosurgery, Loma Linda University School of Medicine, Loma Linda, California 92350, USA

4 Department of Anesthesiology, Loma Linda University School of Medicine, Loma Linda, California 92350, USA

5 Department of Physiology and Pharmacology, Loma Linda University, Risley Hall, Room 219, 11041 Campus Street, Loma Linda, California 92354, USA 


\section{Introduction}

Spontaneous intracerebral hemorrhage (ICH) is a lifethreatening stroke subtype accounting for $10-15 \%$ of stroke that lacks effective drug therapy and has high mortality and morbidity [1-3]. The mass effect of the hematoma and toxic substances released from the erythrocytes lysis are crucial factors leading to neuronal death, delayed cerebral edema, and poor prognosis after ICH $[4,5]$. Both clinical trials on surgical removal of hematoma and experimental studies suggest that efficient removal of the hematoma is an effective therapeutic target in ICH [6-8]. However, increasing evidences from clinical trials indicate that open surgical hematoma evacuation did not improve patient outcomes; therefore, endogenous mechanisms of hematoma resolution need to be investigated [9-12].

$\mathrm{C}-\mathrm{C}$ chemokine receptor 4 (CCR4) is a G proteincoupled receptor that was found to be highly expressed in immune cell including microglia, monocytes, and macrophages [13, 14]. Current literature provides evidence that CCR4 has important roles in regulating immune balance and activating innate immune cells in the central nervous system (CNS) $[15,16]$. C-C chemokine ligand 17 (CCL17) is a specific ligand of CCR4, which has been reported to be produced by the murine brain hippocampal CA1 neurons [17]. CCL17 deficiency resulted in a diminished microglia density under homeostatic and inflammatory conditions [17]. CCL17 is recognized as a homeostatic and inducible neuromodulatory chemokine which is required to maintain the typical, highly branched morphology of hippocampal microglia under homeostatic conditions, and promotes the adaptation of microglial morphology to acute LPS-induced neuroinflammation [17, 18]. Extracellular signalregulated kinase (ERK) $1 / 2$, a prototypic subfamily of MAPKs, has been shown to be the downstream protein after CCR4 activation [19]. Activated CCR4 can promote ERK phosphorylation and activate the downstream pathway. Previous studies showed that ERK increased the expression of nuclear factor erythroid-related factor 2 (Nrf2), and Nrf2 has been shown to enhance hematoma resolution via increasing CD163 in macrophages $[20,21]$. However, the expression and immune function of CCR4 in the CNS after experimental ICH have not been systemically studied.

Hemoglobin (Hb) scavenger receptor CD163 [22] was found to be increased over time in parallel with hematoma resolution after blood-induced ICH in piglets [23]. The haptoglobin-CD163 scavenging system played a critical role in endogenous elimination of blood metabolites from the ICHaffected brain; thus, it can potentially reduce primary and secondary injury after ICH and improve prognosis [24]. Hence, promoting CD163 expression on microglia and macrophages may be a potentially effective method for the treatment of
ICH. In this study, we aimed to evaluate whether recombinant CCL17 (rCCL17) treatment can promote hematoma resolution through CCR4/ERK/Nrf2/CD163 pathway thereby improving short- and long-term neurological outcomes after $\mathrm{ICH}$ in mice.

\section{Materials and Methods}

\section{Animals}

The study was conducted following the guidelines of the National Institutes of Health for the care and use of laboratory animals. The experiment protocol was approved by Loma Linda University Animal Care and Use Committee. Mice were housed in a temperature and humidity controlled environment and had free access to water and food. The light in the room was controlled in a 12-h light/dark cycle. Mice were randomly assigned to each group and SPSS version 18.0 software-generated random numbers were used with a unique code linking to the individual animal.

\section{Experimental Design}

The following four separate experiments and the summary of experimental groups are shown in Fig. 1. Histological images and functional outcomes were assessed in a blinded manner. The investigators were blinded for the surgical procedures and treatment. S.X.D. performed surgeries, and P.J. and Z.T. did all other measurements.

\section{Experiment 1}

To study the expression of endogenous CCL17, CCR4, Nrf2, and $\mathrm{CD} 163$ after ICH, 42 mice were randomly divided into 7 groups: sham, ICH after 6 h, 12 h, 24 h, 72 h, 5 days, and 7 days ( $n=6$ /group) for western blot. Additional 4 mice were used for immunofluorescence (IF) staining to localize CCR4 on microglia in 72-h post-ICH and in sham groups ( $n=2$ /group).

\section{Experiment 2}

To determine the best treatment dosage for rCCL17, 30 mice were randomly assigned to 5 groups for neurobehavior evaluation ( $n=6$ /group): sham, ICH + vehicle (10\% DMSO dissolved in corn oil), ICH + rCCL17 $(10 \mu \mathrm{g} / \mathrm{kg}), \mathrm{ICH}+\mathrm{rCCL} 17$ $(30 \mu \mathrm{g} / \mathrm{kg}), \mathrm{ICH}+\mathrm{rCCL} 17(90 \mu \mathrm{g} / \mathrm{kg})$. rCCL17 was administered intranasally (i.n.) at $1 \mathrm{~h}$ following ICH. Neurobehavioral functions (Corner Turn, Modified Garcia and Forelimb Placement Tests) were evaluated at 24-h and 72-h post-ICH. Based on the results of the neurological tests, additional 102 mice were randomly assigned to sham, vehicle, and rCCL17 $(30 \mu \mathrm{g} / \mathrm{kg}$ ) groups to observe the effect of rCCL17 on brain 
Experiment 1: Time course of endogenous CCL17, CCR4, Nrf2, CD163 and cellular localization of CCR4 after ICH.

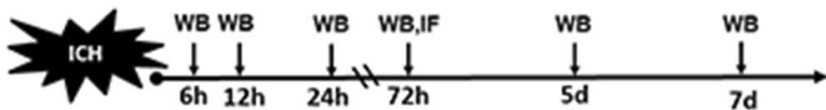

Experiment 2: The effects of rCCL17 treatment on short-term neurobehavior, hematoma volume, hemoglobin content, brain edema after ICH.

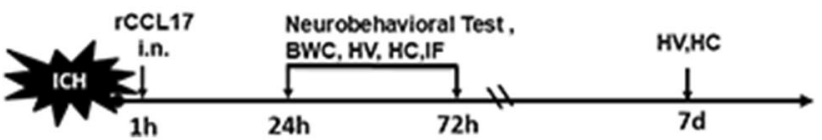

Experiment 3: The effects of rCCL17 treatment on long-term neurobehavior after ICH.

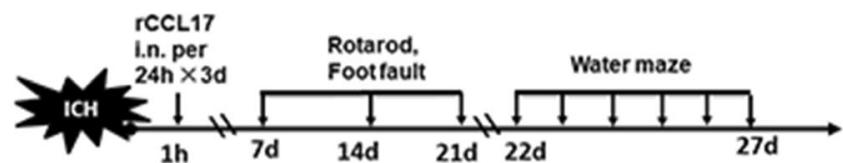

Groups(WB,If):

(1) Sham

(2) $\mathrm{ICH} 6 \mathrm{~h}$

(3) ICH $12 \mathrm{~h}$

(4) ICH $24 \mathrm{~h}$

(5) ICH $72 \mathrm{~h}$

(6) ICH Sd

(7) $1 \mathrm{CH} 7 \mathrm{~d}$

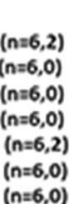

Groups (Short-term neurobehavior, BWC, HV, IF ):

(1) Sham

(n=6,12,6/time point, 4)

(2) $\mathrm{ICH}+$ Vehicle $\quad(n=6,12,6 /$ time point,4)

(3) $\mathrm{ICH}+\mathrm{rCCl} 17$ (10ug/kg) (n=6, 0, 0/time point, 0)

(4) $1 \mathrm{CH}+\mathrm{rCCL} 17$ (30ug/kg) (n=6, 12,6/time point,4)

(5) $1 \mathrm{CH}+\mathrm{rCCl}_{17}$ (90ug/kg) (n=6, 0,0/time point, 0)

Mortality
0
0
0
0
0
0
0

Mortality

0 5 0

\begin{tabular}{|c|c|c|}
\hline \multirow{2}{*}{\multicolumn{2}{|c|}{$\begin{array}{l}\text { Groups (long-term neurobehavior): } \\
\text { (1) Sham } \\
(n=8)\end{array}$}} & \multirow{2}{*}{$\begin{array}{c}\text { Mortality } \\
0\end{array}$} \\
\hline & & \\
\hline (2) ICH + Vehicle & $(n=8)$ & 0 \\
\hline (3) $1 \mathrm{CH}+r \mathrm{CCl} 17$ (30ug/kg) & $(n=8)$ & 0 \\
\hline
\end{tabular}

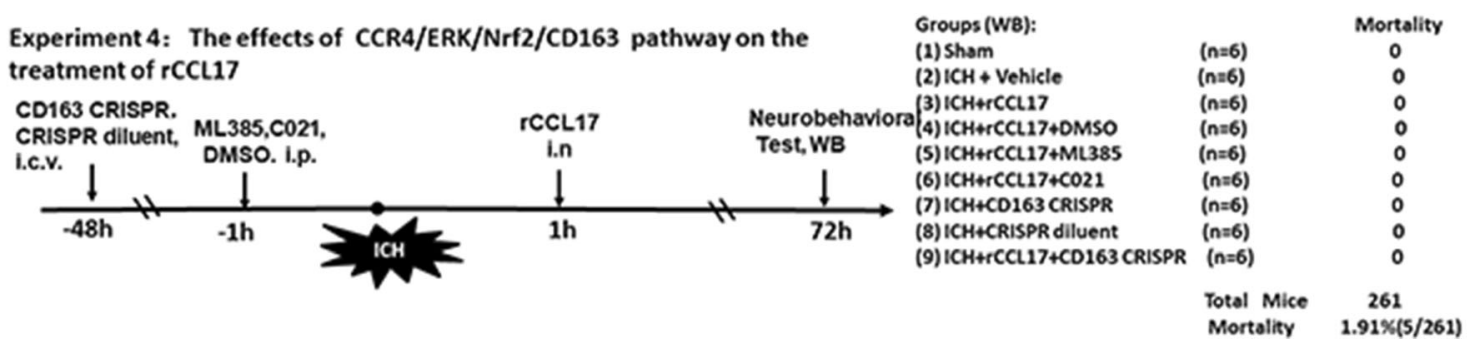

Fig. 1 Experimental design and animal groups. ICH, intracerebral hemorrhage; CCL17, C-C chemokine ligand17; rCCL17, recombination C-C chemokine ligand17; CCR4, C-C chemokine receptor 4; Nrf2, nuclear factor erythroid 2-related factor 2; WB, western blot; IF,

edema and hematoma. Brain water content was assessed at 24$\mathrm{h}$ and 72 -h post-ICH ( $n=6 /$ group/time point). Hematoma volume and hemoglobin content were measured at days 1,3 , and 7 after ICH ( $n=6 /$ group/time point). Double immunofluorescence staining was performed to localize CD163 with CD11b(a cell surface marker shared by activated microglia/ macrophages and granulocytes) positive cells in the perihematomal region $72 \mathrm{~h}$ after ICH ( $n=4$ /group).

\section{Experiment 3}

To determine the effect of rCCL17 on long-term outcomes after $\mathrm{ICH}, 24$ mice were randomly divided into 3 groups $(n=8 /$ group): sham, ICH + vehicle, ICH + rCCL17 $(30 \mu \mathrm{g} / \mathrm{kg})$. rCCL17 was administered i.n. at 1-h post-ICH and then every $24 \mathrm{~h}$ for three consecutive days. Foot fault test and rotarod test were performed on days 7, 14, and 21 after ICH. Morris water maze was performed on days 22-27 after ICH.

\section{Experiment 4}

To investigate the underlying mechanism of CCR4/ERK/ Nrf2/CD163 pathway, 54 mice were assigned to 9 groups immunofluorescence; HV, hematoma volume; HC, hemoglobin content; BWC, brain water content; CRISPR, clustered regularly interspaced short palindromic repeats; DMSO, dimethyl sulfoxide; i.n., intranasally; i.c.v., intracerebroventricularly

( $n=6$ /group): sham, ICH + vehicle (10\% DMSO dissolved in corn oil $), \mathrm{ICH}+\mathrm{rCCL} 17(30 \mu \mathrm{g} / \mathrm{kg}), \mathrm{ICH}+\mathrm{rCCL} 17$ $(30 \mu \mathrm{g} / \mathrm{kg})+$ DMSO, ICH + rCCL17 $(30 \mu \mathrm{g} / \mathrm{kg})+$ ML385, $\mathrm{ICH}+\mathrm{rCCL} 17(30 \mu \mathrm{g} / \mathrm{kg})+\mathrm{C} 021, \mathrm{ICH}+\mathrm{CD} 163$ CRISPR Knockout, ICH + CRISPR diluent, ICH + rCCL17 $(30 \mu \mathrm{g} / \mathrm{kg})$ + CD163 CRISPR Knockout. The CCR4 inhibitor C021 and Nrf2 inhibitor ML385 was administered intraperitoneal (i.p) at $1 \mathrm{~h}$ prior to ICH. CD163 CRISPR Knockout was injected intracerebroventricular (i.c.v) route at $48 \mathrm{~h}$ prior to $\mathrm{ICH}$. Neurobehavioral functions were evaluated and the expressions of CCL17, CCR4, p-ERK, ERK, Nrf2, and CD163 were detected by western blot at 72-h post-ICH.

\section{ICH Model}

The autologous blood injection ICH model was induced as previously described [25]. In brief, mice were anesthetized with a mixture of ketamine $(100 \mathrm{mg} / \mathrm{kg})$ and xylazine $(10 \mathrm{mg} / \mathrm{kg})$. Then, mice were fixed on the stereotactic frame (Kopf Instruments, Tujunga, CA) in a prone position. According to the established coordinates $(0.2 \mathrm{~mm}$ anterior and $2.3 \mathrm{~mm}$ right lateral from bregma), a $1-\mathrm{mm}$ cranial burr hole was made. A total of $30 \mu \mathrm{L}$ autologous blood collected 
from the rodent's femoral artery was instilled through the burr hole using a 1-mL syringe. First, the syringe was advanced to a depth of $3.0 \mathrm{~mm}$ below the dura and $5 \mu \mathrm{L}$ of blood was delivered at a rate of $3 \mu \mathrm{L} / \mathrm{min}$ using a microinjection pump (Harvard Apparatus, Holliston, MA). This was followed by a waiting period of $5 \mathrm{~min}$ after which the needle was lowered to a depth of $3.5 \mathrm{~mm}$, and $25 \mu \mathrm{L}$ of blood was delivered to the right striatum. In order to minimize blood backflow along the needle tract, the needle was kept in place for 10 min after the completing the injection of autologous blood. Sham surgery consisted of needle insertion but without injection of blood. Rectal temperature was maintained at $37.0 \pm 0.5{ }^{\circ} \mathrm{C}$ by a feedback-controlled rodent surgical heating pad (Gaymar, Orchard Park, NY).

\section{Drug Administration}

Recombinant CCL17 (rCCL17) (14013, LSBio, WA) at dosages of 10,30 , and $90 \mu \mathrm{g} / \mathrm{kg}$ dissolved in $5 \%$ dimethyl sulfoxide (DMSO) was administered i.n. at $1 \mathrm{~h}$ after ICH induction as previously reported [26]. Under anesthesia, mice were placed in a supine position and rCCL17 dissolved in DMSO or DMSO was administered alternating between the left and right nares. A total volume $20 \mu \mathrm{L}$ of rCCL17 was administered i.n. with one drop ( $5 \mu \mathrm{L} /$ drop) every 5 min alternating between the two nares over a period of $20 \mathrm{~min}$. CCR4 inhibitor C021 dihydrochloride (C021) (3581, Tocris, USA) (1 mg/kg) [27] and Nrf2 inhibitor ML385 (6887, Tocris, USA) (30 mg/kg) [28] dissolved in 5\% DMSO was intraperitoneally administered $1 \mathrm{~h}$ before ICH induction. According to the instruction, CD163 CRISPR knockout (sc-429989, Santa Cruz Biotechnology, USA) plasmids (r) and CRISPR diluent was administered by i.c.v. route $48 \mathrm{~h}$ prior to $\mathrm{ICH}$ induction. The i.c.v. injection was performed at a rate of $0.25 \mu \mathrm{L} / \mathrm{min}$ at the following coordinates from bregma: $0.3 \mathrm{~mm}$ posterior, $1 \mathrm{~mm}$ right lateral, and $2.3 \mathrm{~mm}$ ventral.

\section{Assessment of Short-Term Neurobehavioral Deficits}

The modified Garcia test, corner turn test, and the forelimb placement test were performed as previously published from our laboratory [29] to evaluate acute neurological deficits at $24 \mathrm{~h}$ and $72 \mathrm{~h}$ after ICH in a blinded fashion. The modified Garcia test with a score ranging from 0 to 21 included 7 tests: spontaneous activity, axial sensation, vibrissae proprioception, limb movement, lateral turning, forelimb walking, and climbing. Each individual test were given scores from 0 to 3 $(0=$ worst; 3 = best $)$. For the forelimb placement test, the left forelimb placement was calculated as left forelimb placement/ (left forelimb placement + right forelimb placement $) \times 100 \%$. For the corner turn test, mice were recorded for a total of 10 trials and a score was calculated as the number of left turns/10 trials $\times 100 \%$.

\section{Long-Term Neurobehavioral Assessment}

Foot fault and rotarod tests were conducted at days 7, 14, and 21 post-ICH. The water maze tests was performed at days $22-$ 27 after ICH as previously published from our lab [30]. The swim distance and escape latency during days 1 to 5 of water maze testing and probe test on day 6 of testing were recorded.

\section{Brain Water Content Measurement}

The wet/dry method was used to measure brain water content as previously reported [31]. Under deep anesthesia, mice were euthanized and the brains were immediately taken out. Each brain sample was divided into five parts: ipsilateral and contralateral cortex, ipsilateral and contralateral basal ganglia, and cerebellum. Each part was weighed immediately on an analytical microbalance to get the wet weight (WW). Then the samples were dried at $100{ }^{\circ} \mathrm{C}$ for $48 \mathrm{~h}$ to get the dry weight (DW). Brain water content $(\%)$ was calculated as (WW$\mathrm{DW}) / \mathrm{WW} \times 100 \%$.

\section{Hematoma Volume and Hemoglobin Content Measurement}

Hematoma volume measurement was performed as previously reported from our lab [32] at $24 \mathrm{~h}, 72 \mathrm{~h}$, and 7 days after ICH induction. In brief, under deep isoflurane anesthesia mice were transcardially perfused with $4{ }^{\circ} \mathrm{C}$ phosphate-buffered saline (PBS). The whole brain was taken out and cut into 1$\mathrm{mm}$ coronal section to take a picture. Hematoma volume was calculated using the following formula: hematoma volume $\left(\mathrm{mm}^{3}\right)=$ area of every section $\left(\mathrm{mm}^{2}\right) \times$ section number $\times$ thickness $(\mathrm{mm})$ by the Image J software.

Hemoglobin content was evaluated to quantify hematoma at the same points as hematoma volume measurement. Hemoglobin assay was performed using Drabkin's reagent as previously published [33]. The brain samples which were obtained for hematoma volume assessment were used. The brain samples were put into glass tubes with $3 \mathrm{~mL}$ of PBS. The samples were homogenized for $60 \mathrm{~s}$ followed by ultrasonication for $1 \mathrm{~min}$ to lyse the erythrocyte membranes. The homogenized samples were centrifuged $\left(12,000 \mathrm{rcf}, 4^{\circ} \mathrm{C}\right)$ for $30 \mathrm{~min}$. The supernatant was obtained and Drabkin's reagent (Sigma-Aldrich, USA) was added at a 4:1 ratio and allowed to react for $15 \mathrm{~min}$. The hemoglobin absorbance was measured by a spectrophotometer at $540 \mathrm{~nm}$ (Genesis 10uv; Thermo Fisher Scientific, USA). The hemoglobin content was calculated as a ratio compared with sham group.

\section{Western Blot}

Western blot was conducted as previously reported [34]. Briefly, at the respective time-points after $\mathrm{ICH}$, under deep 
isoflurane anesthesia, mice were transcardially perfused with cold PBS, and brains were removed and separated into the two hemispheres. The ipsilateral brain hemisphere was homogenized in RIPA lysis buffer (sc-24948, Santa Cruz Biotechnology, USA) and then centrifuged at $14000 \mathrm{rpm}$ for $30 \mathrm{~min}$. Protein samples $(4 \mu \mathrm{L}, 1.5 \mathrm{mg} / \mathrm{mL})$ were added to a $10 \%$ SDS-PAGE gel. After gel electrophoresis and transfer to a nitrocellulose membrane, membranes were blocked with $5 \%$ nonfat blocking grade milk (Bio Rad Laboratories, USA) for $2 \mathrm{~h}$. The membranes were incubated overnight at $4{ }^{\circ} \mathrm{C}$ with the primary antibodies: rabbit anti-CCL17 (1:1000, ab182793, Abcam, USA); rabbit anti-CCR4 (1:1000, GTX53474, Gene Tex, USA); rabbit anti-Nrf2 (1:1000, GTX103322, Gene Tex); mouse anti-p-ERK (1:1000, Santa Cruz Biotechnology, USA); mouse anti-ERK (1:1000, sc-514302, Santa Cruz Biotechnology, USA); mouse anti-CD163 (1:1000, GTX42366, Gene Tex). The membranes were incubated with mouse anti- $\beta$ actin antibody (1:5000, Abcam, USA) as a loading control. The relative density of the bands was quantified as a ratio of the target band intensity to the band intensity of $\beta$ actin by ImageJ software.

\section{Immunohistochemistry}

Immunofluorescence staining was performed at $72-\mathrm{h}$ postICH. The mice were anesthetized and transcardially perfused with PBS followed by $4 \%$ paraformaldehyde as previously described [35]. The brains were fixed with formalin for 2 days followed by dehydration with $30 \%$ sucrose solution for 3 days. Brain samples were prepared in $10-\mu \mathrm{m}$ coronal section using a cryostat (CM3050S, Leica Biosystems, USA). The sections were embedded overnight at $4{ }^{\circ} \mathrm{C}$ with the following primary antibodies: anti-CCR4 (1:100, Santa Cruz Biotechnology, USA); anti-CD11b (1:200, Abcam, USA) and anti-CD163 (1:100, Santa Cruz Biotechnology, USA). The slides were incubated with appropriate secondary antibodies (1:200, Jackson ImmunoResearch, USA) for $2 \mathrm{~h}$ at room temperature followed by applying DAPI. Stained brain sections were observed using a DMi8 fluorescent microscope (Leica Microsystems, USA) under a $400 \times$-fold field. The number of CD11b- (a cell surface marker shared by activated microglia/macrophages and granulocytes) positive cells costained with CD163 was counted from four fields in the perihematomal area on each of the three sections per animal. The number of CD11b and CD163 co-stained positive cells was averaged to provide a single value for each mouse.

\section{Statistical Analysis}

Parametric data was presented as mean \pm standard deviation of the mean (SD). Western blot data were analyzed using oneway ANOVA followed by Tukey post hoc test. Behavior data were analyzed using two-way repeated measure ANOVA and
Tukey post hoc test. The cell counting data was analyzed using chi-square test. It was considered as statistically significant if $P$ values were $<0.05$. All the data were graphed and analyzed by GraphPad Prism 7 (La Jolla, CA, USA).

\section{Results}

\section{Animal Mortality Rate}

A total of 261 male CD-1 mice (weight: 30-40 g; Charles River Laboratory, Wilmington, MA, USA) were used for the study. Two mice died due to deep anesthesia before ICH surgery and three mice died $1 \mathrm{~h}$ after ICH surgery. The mortality was $1.915 \%$. There was no difference in the number of deaths of mice between the ICH groups.

\section{Endogenous CCL17, CCR4, Nrf2, and CD163 Expression Increased Post-ICH}

Western blot results showed the expression of endogenous CCL17 was increased after ICH from $6 \mathrm{~h}$ reaching a peak at 1 to 5 days compared with sham group ( $p<0.05$, Fig. 2A, B). CCR4 significantly increased from $6 \mathrm{~h}$ to 7 days after ICH compared with sham $(p<0.05$, Fig. 2A, C). Nrf2 increased after ICH from $6 \mathrm{~h}$ to 7 days with a peak at $72 \mathrm{~h}$ compared with sham group ( $p<0.05$, Fig. 2A, D). Accordingly, CD163, the products of Nrf2 activation, was significantly increased at $6 \mathrm{~h}$ after ICH and peaked at $24 \mathrm{~h}$ then started to decline $(p<0.05$, Fig. 2A, E). Immunofluorescence staining demonstrated that CCR4 was co-localized on activated microglia (CD11b, a cell surface marker shared by microglia/macrophages and granulocytes) in the perihematomal region at 72-h post-ICH (Fig. 2F).

\section{rCCL17 Improved Short-Term Neurobehavior and Reduced Brain Edema at $\mathbf{7 2} \mathrm{h}$ After ICH}

The neurobehavioral score was significantly decreased in all ICH groups compared to sham at 24-h and 72-h post-ICH ( $p<0.05$, Fig. 3A-C). Intranasal administration of rCCL17 (30 $\mu \mathrm{g} / \mathrm{kg}$ and $90 \mu \mathrm{g} / \mathrm{kg}$ ) significantly improved neurological function at 72-h post-ICH than $\mathrm{ICH}+$ vehicle group $(p<0.05$, Fig. 3A-C). Similarly, the brain water content in ipsilateral regions was increased at $24 \mathrm{~h}$ and $72 \mathrm{~h}$ after $\mathrm{ICH}$ compared with sham group ( $p<0.05$, Fig. 3D-E). Intranasal administration of rCCL17 $(30 \mu \mathrm{g} / \mathrm{kg})$ significantly reduced brain water content in the ipsilateral basal ganglia and cortex compared with $\mathrm{ICH}+$ vehicle group at 72 -h post-ICH $(p<0.05$, Fig. $3 \mathrm{E})$. Based on the neurobehavioral and brain water content results, the medium dosage of rCCL17 $(30 \mu \mathrm{g} / \mathrm{kg})$ was selected for further research. 


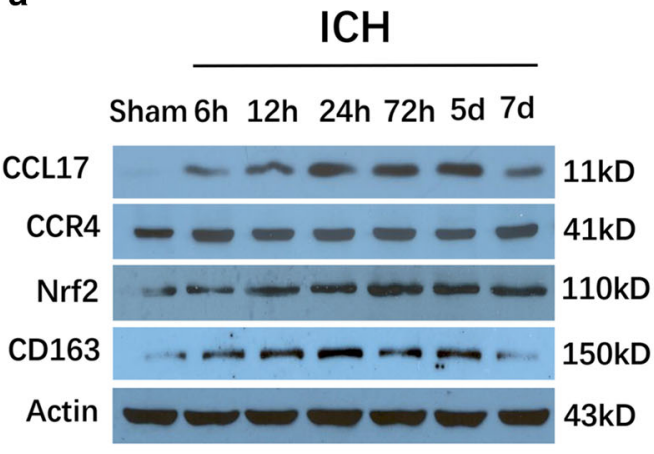

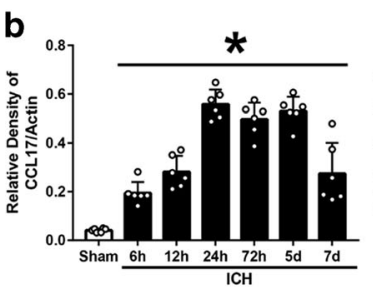
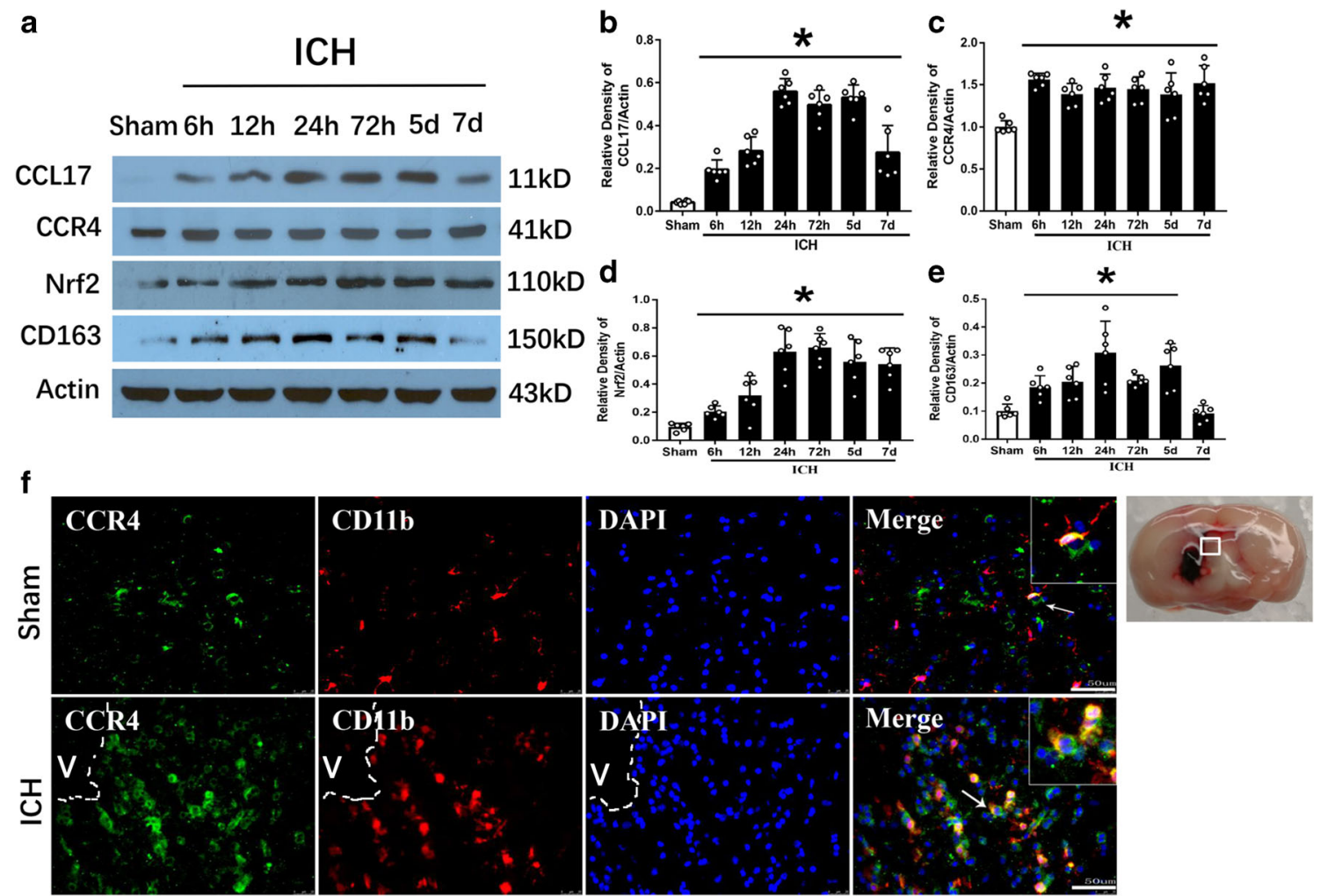

Fig. 2 The time course of endogenous CCL17, CCR4, Nrf2, and CD163 expressions after ICH. (A) Representative WB bands and (B-E) quantitative analysis of CCL17, CCR4, Nrf2, and CD163 expressions, respectively after ICH. $* p<0.05$ versus sham, mean $\pm \mathrm{SD}$, one-way ANOVA, Tukey test, $n=$ 6/group. (F) Brain sample with schematic illustration showing one areas in the perihematomal region (indicated by white box) from where the images were

taken for immunofluorescence staining (pictures are shown in panel F), colocalization of CCR4 (green) with CD11b (red) at $72 \mathrm{~h}$ after sham-operation or ICH in the perihematomal area (v representing the hematoma area). Nuclei were stained with DAPI (blue), scale bar $=50 \mu \mathrm{m}, n=2$ /group. DAPI, 4',6diamidino-2-phenylindole
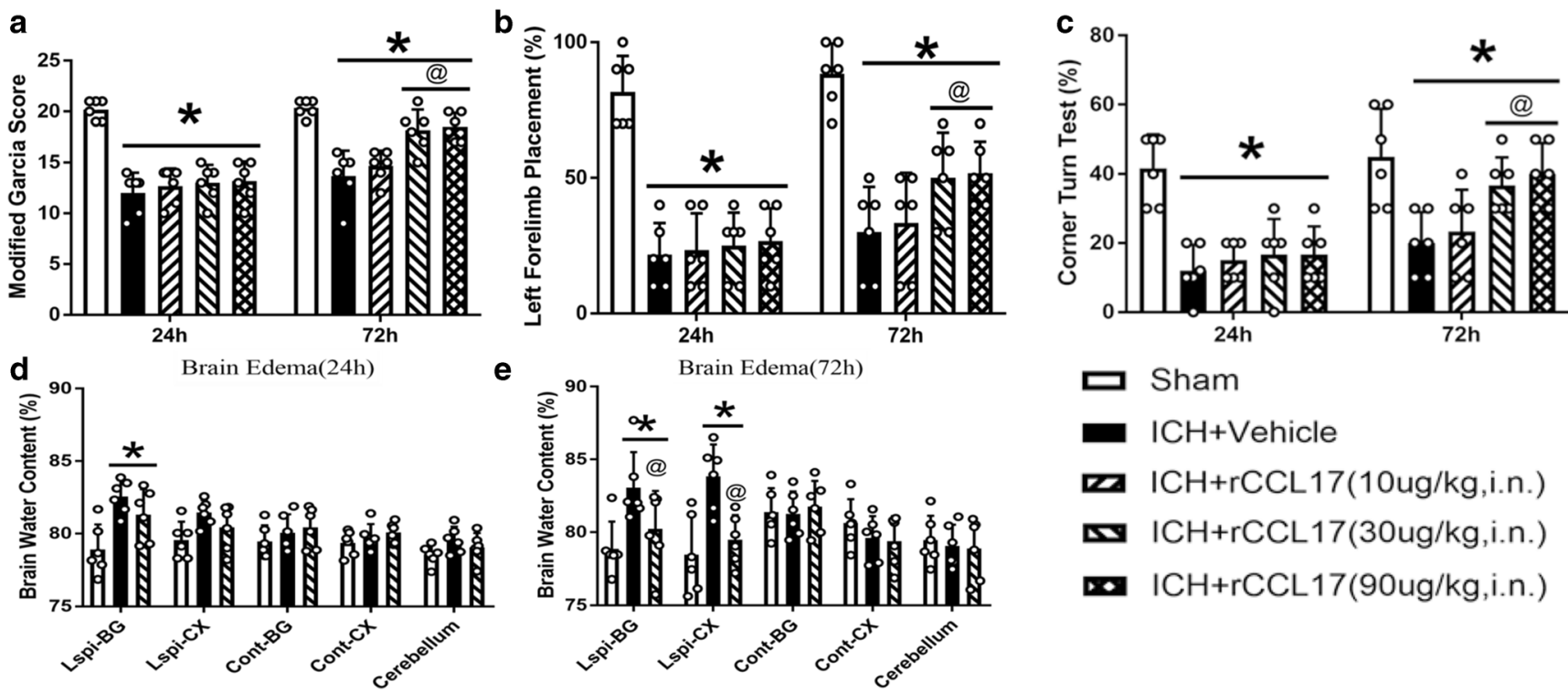

Fig. 3 Intranasal administration of rCCL17 improved short-term neurological function and reduced brain edema at $72 \mathrm{~h}$ after ICH. (A) Modified Garcia test; (B) left forelimb placement test; (C) corner turn test; (D) brain edema at 24-h post-ICH; (E) brain edema at 72-h post-ICH. Short-term neurological results showed that rCCL17 treatment at the dosage of 30

and $90 \mu \mathrm{g} / \mathrm{kg}$ improved short-term neurological function significantly, compared to vehicle and low dosage groups. So, we further chose the middle dosage for brain edema measurements. ${ }^{*} p<0.05$ versus sham, $@ p<0.05$ versus $\mathrm{ICH}+$ vehicle, mean $\pm \mathrm{SD}$, two-way repeated measures ANOVA (A, B, C), One-way ANOVA (D, E), Tukey test, $n=6 /$ group 


\section{rCCL17 Promoted Hematoma Resolution and Increased the Expression of CD163 on Microglia/Macrophages $72 \mathrm{~h}$ after ICH}

To evaluate the effect of rCCL17 on hematoma resolution, mice brains were sectioned at $24 \mathrm{~h}, 72 \mathrm{~h}$, and 7 days after $\mathrm{ICH}$. The brain samples from sham group showed only the needle track whereas a larger mass of hematoma was observed in $\mathrm{ICH}+$ vehicle and ICH + rCCL17 group at $24 \mathrm{~h}$ after ICH $(p<0.05$; Fig. 4A, B), but there was no statistical difference in hematoma volume between vehicle and rCCL17 treatment group. Although the hematoma volume was smaller in both $\mathrm{ICH}+$ vehicle and $\mathrm{ICH}+\mathrm{rCCL} 17$ treatment group at 72-h and 7-day post-ICH, the hematoma volume was significantly reduced in rCCL17 treatment group compared with vehicle group ( $p<0.05$; Fig. 4A, B). Likewise, the hemoglobin content showed similar tendency after ICH, and hemoglobin content was significantly reduced in rCCL17 treatment group compared with vehicle group ( $p<0.05$; Fig. 4 C). The proportion of CD163-positive activated microglia/macrophages cells was calculated in the perihematomal area (Fig. 4E). At $72 \mathrm{~h}$ after ICH, the number of CD11b-positive activated microglia/ macrophages was increased compared with sham $(p<0.05$; Fig. 4D, F). The rCCL17 treatment group had increased CD163-positive microglia/macrophages cells compared to vehicle group $72 \mathrm{~h}$ after ICH $(p<0.05$; Fig. 4D, F).

\section{rCCL17 Improved Long-Term Neurobehavioral Outcomes After ICH}

Water maze test showed that mice in $\mathrm{ICH}+$ vehicle group had much longer swim distance and escape latency during days 3 to 5 of testing. The rCCL17 treatment group showed improved performance in swim distance and escape latency on days 3 to 5 ( $p<0.05$; Fig. $5 \mathrm{~A}-\mathrm{C})$. The probe trial was conducted on day 6 of water maze testing which showed that the $\mathrm{ICH}+$ vehicle group spent significantly less time in the target quadrant whereas rCCL17-treated mice spent longer duration in the target quadrant compared to the vehicle group (Fig. 5D). Likewise, ICH + vehicle mice displayed significantly
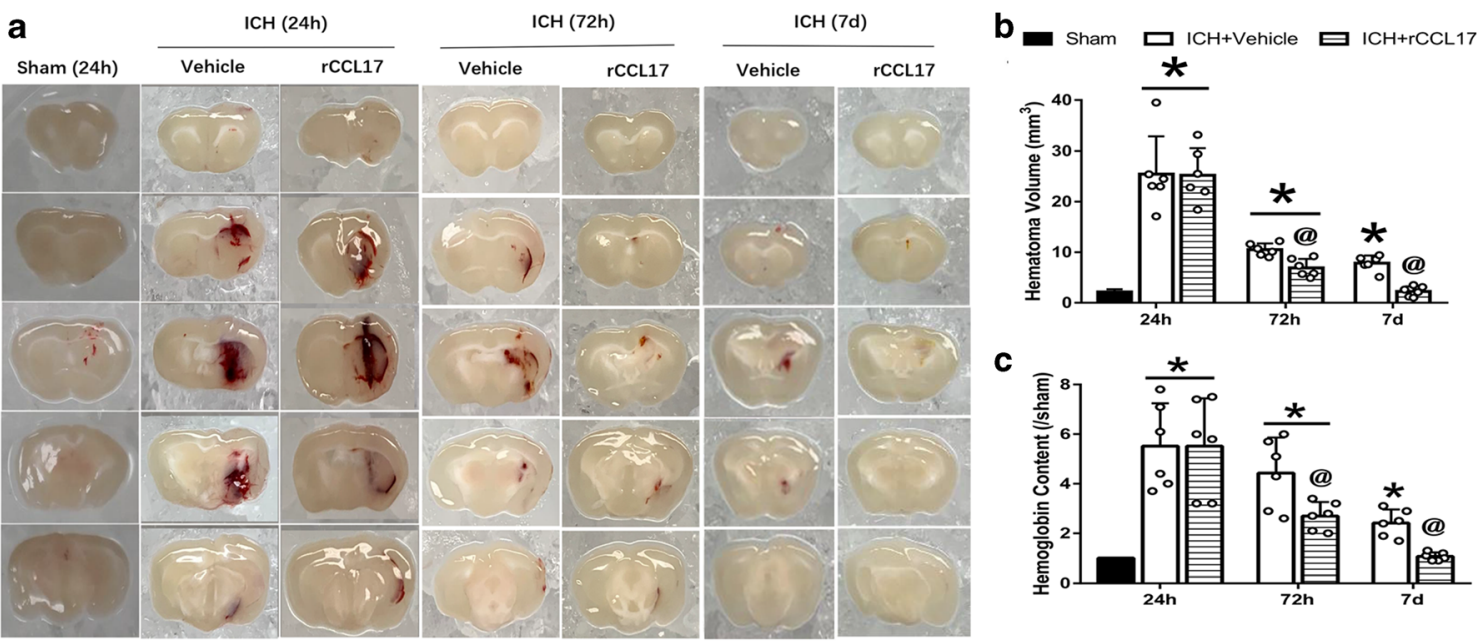

d
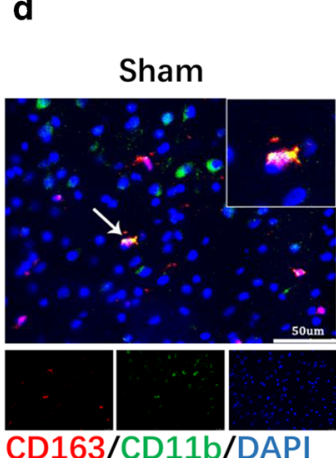

CD163/CD11b/DAPI

$\mathrm{ICH}$

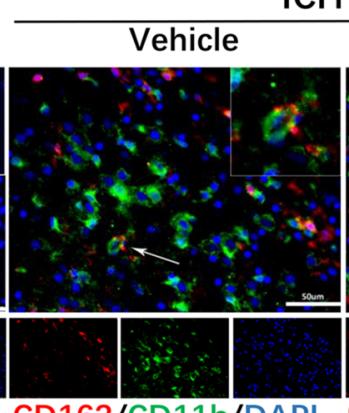

CD163/CD11b/DAPI
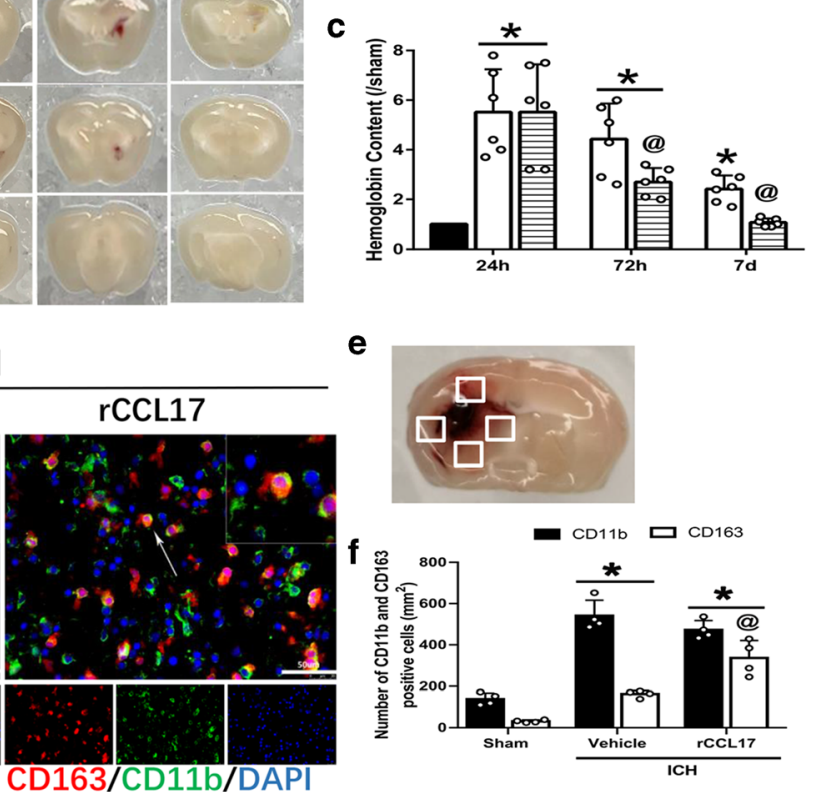

e
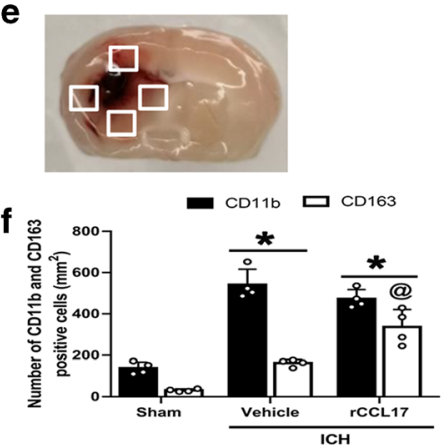

Fig. $4 \mathrm{rCCL} 17(30 \mu \mathrm{g} / \mathrm{kg}$, i.n.) treatment reduced hematoma volume and hemoglobin content at $24 \mathrm{~h}, 72 \mathrm{~h}$, and 7 days after ICH and increased the proportion of CD163-positive cells in activated microglia/macrophages at $72 \mathrm{~h}$ after ICH. (A) Representative photograph of brain sections at $24 \mathrm{~h}$, $72 \mathrm{~h}$, and 7 days after ICH; (B) hematoma volume at $24 \mathrm{~h}, 72 \mathrm{~h}$, and 7-day post-ICH; (C) hemoglobin content at 24-h, 72-h, and 7-day post-ICH. $* p<0.05$ versus sham, @ $p<0.05$ versus $\mathrm{ICH}+$ vehicle, mean $\pm \mathrm{SD}$,

one-way ANOVA, Tukey test, $n=6 /$ group. (D) Immunofluorescence pictures showing co-localization of CD163 with CD11b on experimental group; (E) ICH brain sample showing perihematomal location analyzed for immunostaining; (F) number of CD11b- and CD163-positive cells in microglia/macrophages. $* p<0.05$ versus sham, @ $p<0.05$ versus $\mathrm{ICH}+$ vehicle, $\# p<0.05$ versus $\mathrm{ICH}+\mathrm{rCCL} 17$, mean $\pm \mathrm{SD}$, one-way ANOVA, Tukey test, scale bar $=50 \mu \mathrm{m}, n=4$ /group 

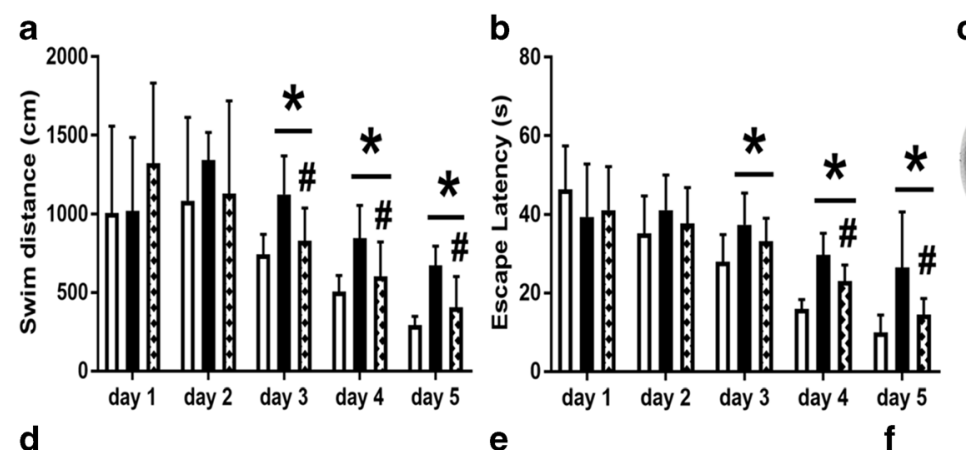

c
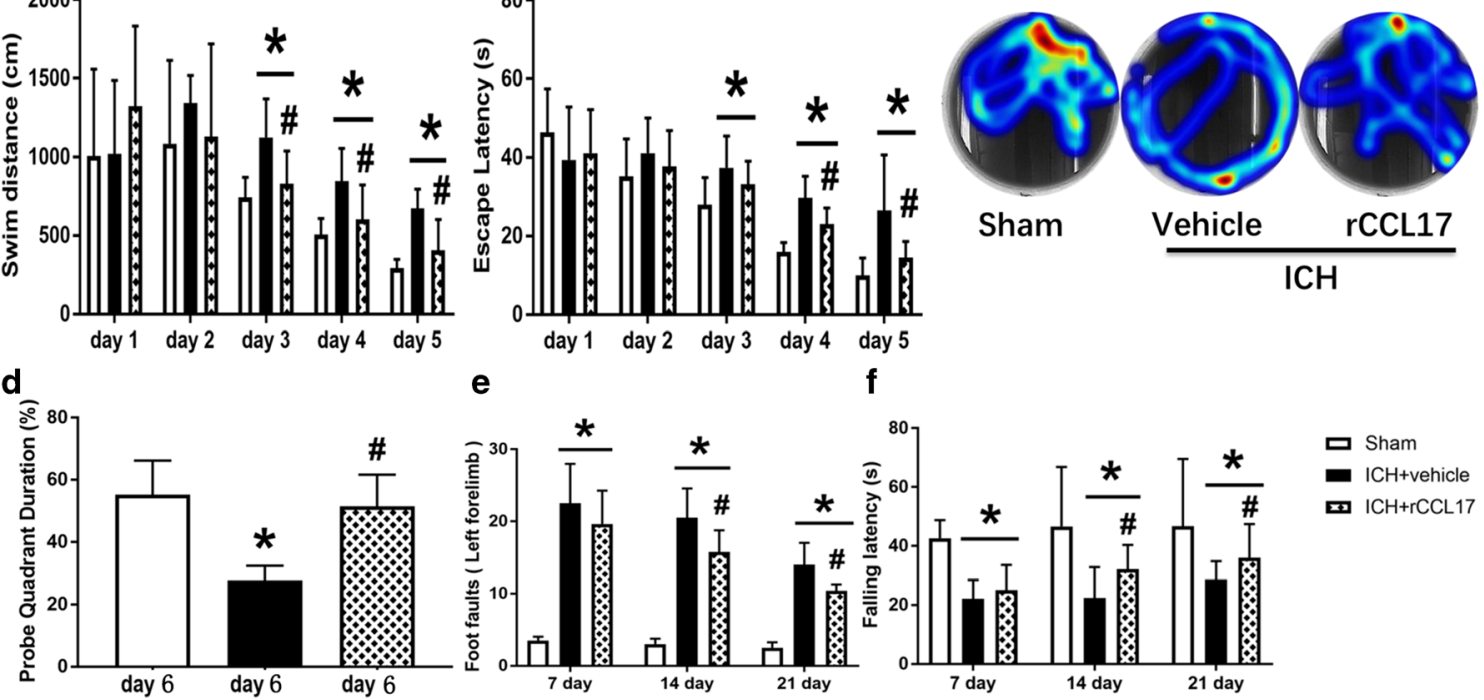

Fig. 5 rCCL17 $(30 \mu \mathrm{g} / \mathrm{kg}$, i.n.) treatment improved long-term motor and memory function after ICH. (A-D) Water Maze tests performed from days 22-27 after ICH, (E) foot fault test, and (F) rotarod test performed at days

7, 14, and 21 after ICH. * $p<0.05$ versus sham, $\# p<0.05$ versus $\mathrm{ICH}+$ vehicle, mean $\pm \mathrm{SD}$, two-way repeated measures ANOVA (A, B), oneway ANOVA for (D, E, F), Tukey test, $n=8 /$ group

increased left forelimb foot faults compared with sham group, and $\mathrm{ICH}+$ vehicle mice had shorter falling latency than sham group in the rotarod test performed at days 7, 14, and 21 after ICH. Mice in rCCL17 treatment group showed significant improvement in both tests ( $p<0.05$; Fig. 5E, F).

\section{rCCL17 Increased CD163 Expression Via Activation of CCR4/ERK/Nrf2 Pathway at $72 \mathrm{H}$ After ICH}

The expression of CCL17 increased after ICH and was further increased with the administration of rCCL17 compared to vehicle group ( $p<0.05$; Fig. 6A). The expression of CCL17 did not change with the administration of CCR4 inhibitor C021 ( $p<0.05$; Fig. 6A, B). CCR4 expression increased after ICH compared to sham, and it was decreased with $\mathrm{C} 021$ administration in the $\mathrm{ICH}+\mathrm{rCCL} 17+\mathrm{C} 021$ group compared to $\mathrm{ICH}+$ rCCL17 group $(p<0.05$; Fig. 6A, C). The expression of p-ERK, Nrf2, and CD163 increased with rCCL17 administration in $\mathrm{ICH}+\mathrm{rCCL} 17$ group compared to $\mathrm{ICH}+$ vehicle group ( $p<0.05$; Fig. $6 \mathrm{~A}, \mathrm{D}, \mathrm{E}, \mathrm{F})$. The inhibition of CCR4 by C021 with rCCL17 administration significantly reversed this effect in $\mathrm{ICH}+\mathrm{rCCL} 17+\mathrm{C} 021$ group compared to $\mathrm{ICH}+$ rCCL17 group ( $p<0.05$; Fig. 6A, D, E, F).

Neurological function assessed by modified Garcia test, forelimb placement test, and corner turn test showed improvement in $\mathrm{ICH}+\mathrm{rCCL} 17$ group compared to $\mathrm{ICH}+$ vehicle which was reduced in $\mathrm{ICH}+\mathrm{rCCL} 17+\mathrm{C} 021$ group compared to $\mathrm{ICH}+\mathrm{rCCL} 17$ and $\mathrm{ICH}+\mathrm{rCCL} 17+\mathrm{DMSO}$ group $(p<0.05$; Fig. 6G, H, I).

Furthermore, the increased expression of CCR4 after ICH was not changed with the administration of rCCL17 and Nrf2 inhibitor ML385 (Fig. 7A, B). The expression of p-ERK increased in $\mathrm{ICH}+\mathrm{rCCL} 17$ group compared to $\mathrm{ICH}+$ vehicle, and there was no effect of ML385 on p-ERK expression $(p<0.05$; Fig. 7A, C). Nrf2 and CD163 expression increased with the administration of rCCL17 in the ICH + rCCL17 group compared to $\mathrm{ICH}+$ vehicle group, and this effect was reversed by ML385 in ICH + rCCL17 + ML385 group $(p<0.05$; Fig.7A, $\mathrm{D}, \mathrm{E})$. Neurological function was reduced in $\mathrm{ICH}+\mathrm{rCCL} 17+$ ML385 group compared to $\mathrm{ICH}+\mathrm{rCCL} 17$ and $\mathrm{ICH}+$ rCCL17 + DMSO group ( $p<0.05$; Fig. 7F, G, H).

In addition, the increased expression of CCR4 after ICH was not changed with the administration of rCCL17 and CD163 CRISPR KO (Fig. 8A, B). The expression of p-ERK and Nrf2 increased in ICH + rCCL17 group compared to $\mathrm{ICH}+$ vehicle, and there was no effect of CD163 CRISPR KO on p-ERK and Nrf2 expression $(p<0.05$; Fig. 8A, C, D). CD163 expression increased after ICH compared to sham, and it was decreased with CD163 CRISPR KO in the ICH + CD163 CRISPR KO group compared to ICH + CRISPR diluent group ( $p<0.05$; Fig. 8A, E). CD163 expression increased with administration of rCCL17 in the $\mathrm{ICH}+\mathrm{rCCL} 17$ group compared to $\mathrm{ICH}+$ vehicle group, and this effect was reversed by CD163 CRISPR KO in ICH + rCCL17 + CD163 CRISPR KO group ( $p<0.05$; Fig. 8A, E). Neurological function was also reduced in $\mathrm{ICH}+\mathrm{CD} 163$ CRISPR KO group and $\mathrm{ICH}+$ rCCL17 + CD163 KO groups compared to $\mathrm{ICH}+\mathrm{rCCL} 17$ and $\mathrm{ICH}+\mathrm{CRISPR}$ diluent group $(p<0.05$; Fig. $8 \mathrm{~F}, \mathrm{G}, \mathrm{H})$.

\section{Discussion}

In the present study, we evaluated the effects of rCCL17 on promoting hematoma resolution after $\mathrm{ICH}$ in a mouse model 

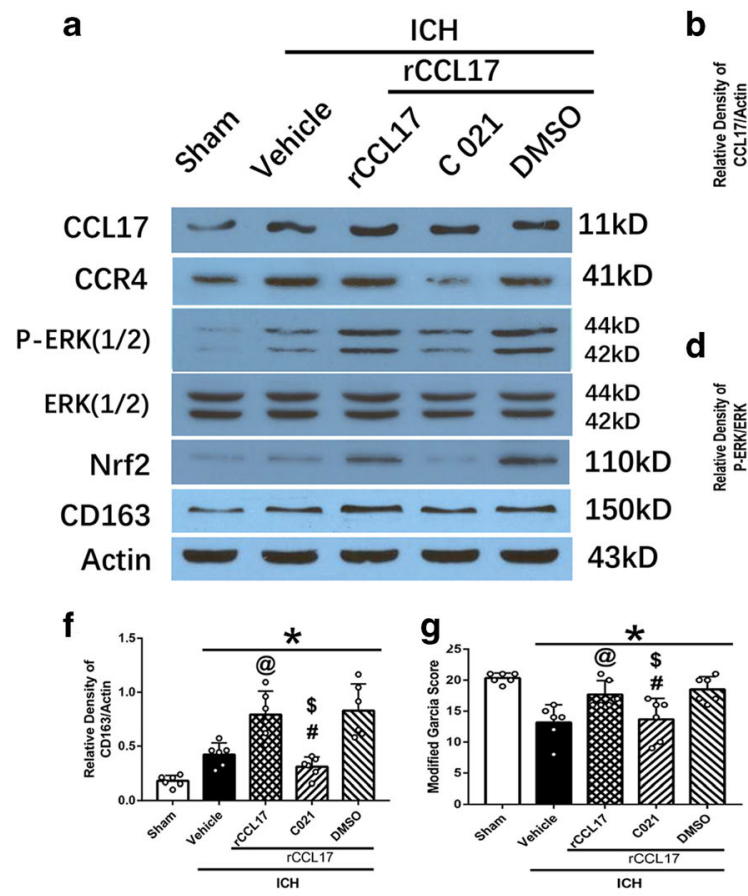

Fig. 6 The effect of rCCL17 activation of CCR4 signaling pathway was reversed by administration of CCR 4 inhibitor, $\mathrm{C} 021$ at $72 \mathrm{~h}$ after ICH. (A) WB bands of CCL17, CCR4, p-ERK1/2, ERK1/2, Nrf2, CD163, and actin; (B-F) quantitative analysis of CCL17, CCR4, p-ERK/ERK, Nrf2, and CD163 expression; $(\mathrm{G})$ modified Garcia test; $(\mathrm{H})$ forelimb placement

and investigated the potential mechanism of the hematoma resolution effects of rCCL17 after ICH. The followings were the novel findings of this study: (a) endogenous expression of CCL17 and its receptors CCR4, Nrf2, and CD163 expressions increased after ICH; (b) CCR4 receptor was expressed on microglia/macrophages; (c) rCCL17 treatment (30 $\mu \mathrm{g} / \mathrm{kg}) \mathrm{im}$ proved short-term and long-term neurological function after ICH in mice; (d) rCCL17 treatment $(30 \mu \mathrm{g} / \mathrm{kg})$ increased CD163 expression in microglia/macrophage, reduced hematoma volume and hemoglobin content at 3 days and 7 days after ICH; (e) rCCL17 treatment (30 $\mu \mathrm{g} / \mathrm{kg})$ promoted hematoma resolution after ICH which was associated with increased CD163 expression through CCR4/ERK/Nrf2 signaling pathway. These findings indicate that rCCL17 administration possibly attenuates hematoma at least in part through CCR4/ERK/Nrf2/CD163 signaling pathway, thereby improving outcomes after ICH.

Current scientific research suggest that the primary adverse effects of the hematoma ("mass effect") and the harmful products of RBCs lysis (hemoglobin and iron) lead to a cascade of events promoting secondary injury which is a crucial factor leading to poor prognosis after ICH $[2,36,37]$. Augmenting the endogenous clearance pathways for red blood cells and promoting microglia mediated phagocytosis have long been proposed as a potential therapeutic strategy for ICH [38].

Microglia, the classical innate immune cells of the brain, are the first non-neuronal cells to respond to brain injury including
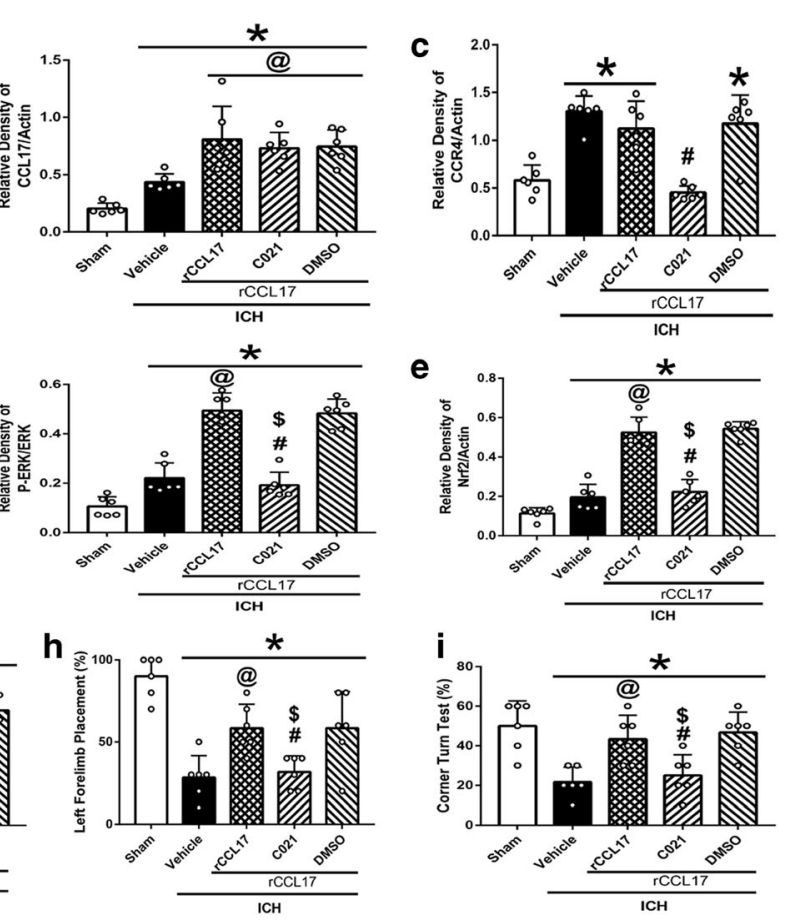

test; (I) corner turn test. * $p<0.05$ versus sham, @ $p<0.05$ versus $\mathrm{ICH}+$ vehicle, $\# p<0.05$ versus $\mathrm{ICH}+\mathrm{rCCL} 17, \$ p<0.05$ versus $\mathrm{rCCL} 17+$ DMSO. Mean $\pm \mathrm{SD}$, one-way ANOVA, Tukey test, $n=6 /$ group. pERK1/2, phosphorylated extracellular regulated protein kinases 1/2; ERK1/2, extracellular regulated protein kinases $1 / 2$

ICH [39], which are capable of damage, repair, cytokine production, and plays an important role in balancing homeostatic stabilization, immune-related, and phagocytic functions within the CNS [40]. Following ICH, activated microglia can differentiate into two phenotypes: M1 (classically activated) or M2 (alternatively activated) and this phenotype changes dynamically [41]. Classically activated M1 microglia phenotype mainly produces pro-inflammatory factors and M2 microglia phenotype are involved in repair through phagocytosis and restrict brain damage [42]. Monocyte-derived macrophages (MDMs) that are derived from peripheral monocyte are recruited in the early hours of $\mathrm{ICH}$, and they mature into macrophages in the CNS parenchyma which contribute to the inflammatory environment. Similar cellular surface markers including CD11b and Iba-1 are expressed by the on brain-derived microglia/macrophages and peripheral macrophages and it is difficult to distinguish them [42]. Both microglia and monocyte-derived macrophages contribute to pro-inflammatory and homeostatic mechanisms within the brain through the secretion of cytokines and other signaling molecules. Furthermore, macrophagocyte that either infiltrate in the injury site or alternatively activated M2-like microglia locally at the peri-hematoma region take part in phagocytosis of red blood cells and removal of tissue debris, a major contribution to hematoma clearance [43].

CD11b, a cell surface marker shared by activated microglia/macrophages and granulocytes, was found to be expressed at $12 \mathrm{~h}$ after experimental intracerebral hemorrhage 


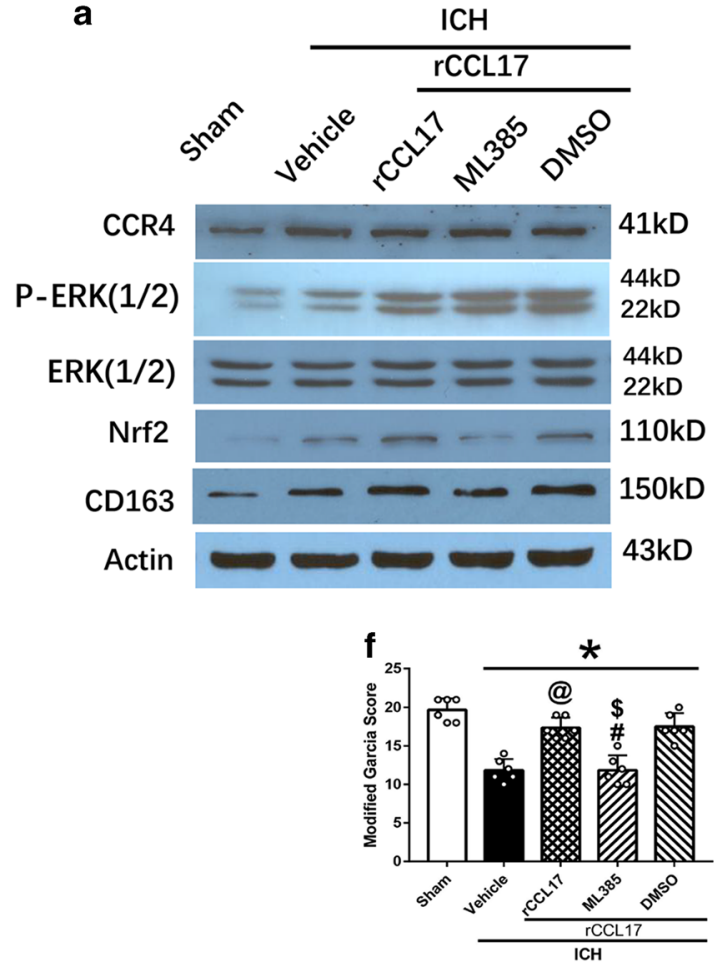

Fig. 7 The effect of rCCL17 was reversed by administration of Nrf2 inhibitor, ML385 at $72 \mathrm{~h}$ after ICH. (A) WB bands of CCR4, p-ERK1/ 2, ERK1/2, Nrf2, CD163, and actin; (B-E) quantitative analysis of CCR4, p-ERK/ERK, Nrf2, and CD163 expression; (F) modified Garcia test; $(\mathrm{G})$ forelimb placement test; $(\mathrm{H})$ corner turn test. * $p<0.05$ versus

[44]. In this study, we used CD11b to localize activated microglia after ICH. We observed that the number of CD11bpositive cells increased in the perihematomal region at $72 \mathrm{~h}$ after ICH. The activation of microglia/macrophages and macrophages from blood may explain the increased number of CD11b-positive cells after ICH, which is consistent with previous study which showed that phagocytic microglia/ macrophages progressively increased in the perihemorrhagic areas for the first week after ICH [45]. However, anti-CD11b antibody cannot distinguish microglia from neutrophils and monocytes infiltrating in the brain after ICH [44]. In the present study, we demonstrated for the first time that intranasal administration of rCCL17 promoted hematoma resolution after ICH and was associated with an increased the proportion of CD163-positive activated microglia/ macrophage in the perihematomal region.

CCL17, originally known as thymus and activationregulated chemokine (TARC), was involved in regulating the trafficking of immune cells such as Treg cells across the blood-brain barrier (BBB) and promoting their transfer to damage sites in multiple sclerosis [16]. Previous study found that the activation and recruitment of Treg cells increased in the brain after ICH which promoted M2-like polarization of microglia and macrophages and contributed to the microglial M1 to M2 phenotype shift [46]. Microglia/macrophages
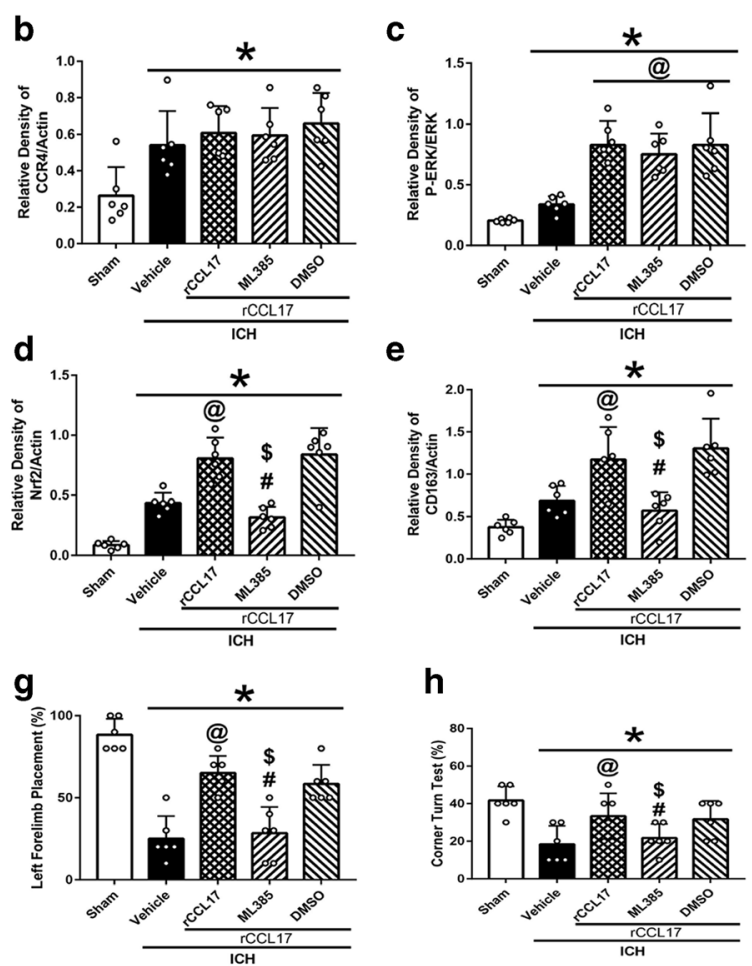

h

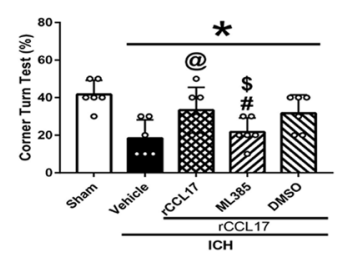

sham, @ $p<0.05$ versus $\mathrm{ICH}+$ vehicle, $\# p<0.05$ versus $\mathrm{ICH}+\mathrm{rCCL} 17$, $\$ p<0.05$ versus rCCL17+DMSO. Mean \pm SD, one-way ANOVA, Tukey test, $n=6$ /group. p-ERK1/2, phosphorylated extracellular regulated protein kinases $1 / 2$; ERK1/2, extracellular regulated protein kinases $1 / 2$

represent the first line of defense against brain injuries. The resident microglia and peripheral macrophages are rapidly mobilized to the site of injury and initiate the release of effector molecules and recruitment of other immune cells. CCR4 was found to be highly expressed on Th2 cells and Treg cells [15]; however, the mechanism linking microglia/macrophage phagocytosis and CCR4 has not been investigated in ICH. In this study, we focused on microglia/macrophage mediated hematoma resolution effects of CCL17, given that hematoma is the primary pathology encountered after ICH which can contribute to the progression of injury. We observed that intranasal administration of rCCL17 promoted hematoma resolution after ICH and increased the expression of the microglia/ macrophage hemoglobin scavenger receptor CD163. Therefore, a mechanism whereby rCCL17 can promote hematoma resolution may involve enhanced microglia/macrophage phagocytosis of blood products in a CCR4-dependent manner. However, further studies are needed to confirm this mechanism, as well as the effects of rCCL17 on the T cell response after ICH.

We observed that endogenous CCL17 increased after ICH in mice. There are some possible explanations for the increased in endogenous CCL17 after ICH: (a) injury and inflammation can stimulate immune cells infiltration in the injury site to secret CCL17; (b) activated microglia in the brain 


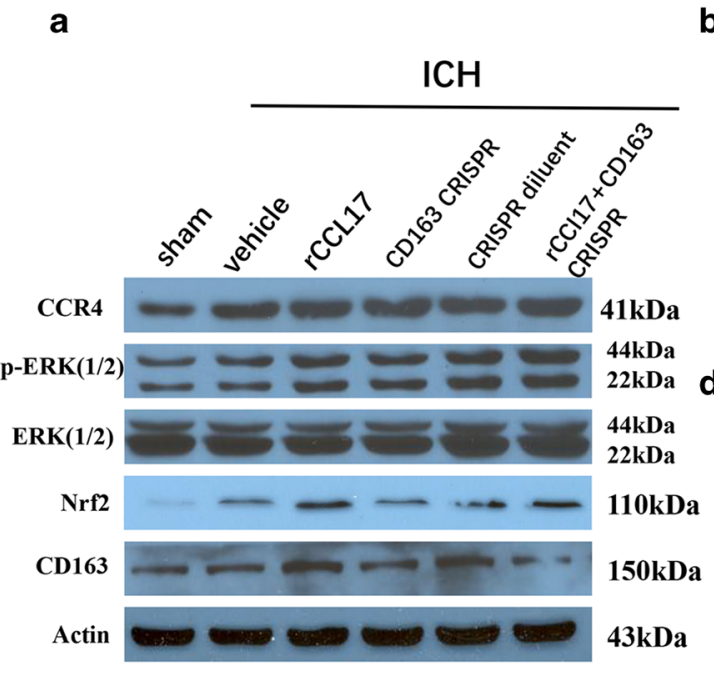

f

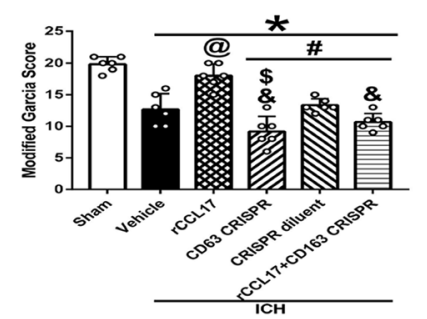

Fig. 8 Administration of CD163 CRISPR knockout prior to ICH abolished the effect of rCCL17 at $72 \mathrm{~h}$ after ICH. (A) WB bands of CCR4, p-ERK1/2, ERK1/2, Nrf2, CD163, and actin; (B-E) quantitative analysis of CCR4, p-ERK/ERK, Nrf2, and CD163 expression; (F) modified Garcia test; $(\mathrm{G})$ forelimb placement test; $(\mathrm{H})$ corner turn test. $* p<0.05$ versus sham, @ $p<0.05$ versus $\mathrm{ICH}+$ vehicle, $\# p<0.05$ versus

producing CCL17; (c) blood circulation brought serum CCL17 which was secreted by peripheral immune cells into brain. Even though the endogenous CCL17 increased after $\mathrm{ICH}$, it may not be sufficient to significantly enhance hematoma clearance after ICH. Our results suggest that exogenous administration of rCCL17 by intranasal route promoted hematoma clearance in ICH mouse model. One of the factors that limit the use of drugs to treat central nervous system disorders is related to the ability of the drug to cross the BBB. Administration of drugs via the nasal route is a non-invasive method that can bypass the BBB for delivering drugs targeted for central nervous system disorders. There is growing scientific support that delivery of drugs via the nasal route may result in higher concentration of drugs to the brain [46]. CCL17 is a small molecule protein, and we chose to administer recombinant CCL17 intranasally in order to deliver it efficiently to the brain bypassing the BBB.

In the present study, we also found $\mathrm{rCCL} 17$ reduced brain edema at $72 \mathrm{~h}$ after ICH and improved short- and long-term neurobehavioral outcomes after ICH. There are some possible explanations for these results: (a) rCCL17 treatment promoted
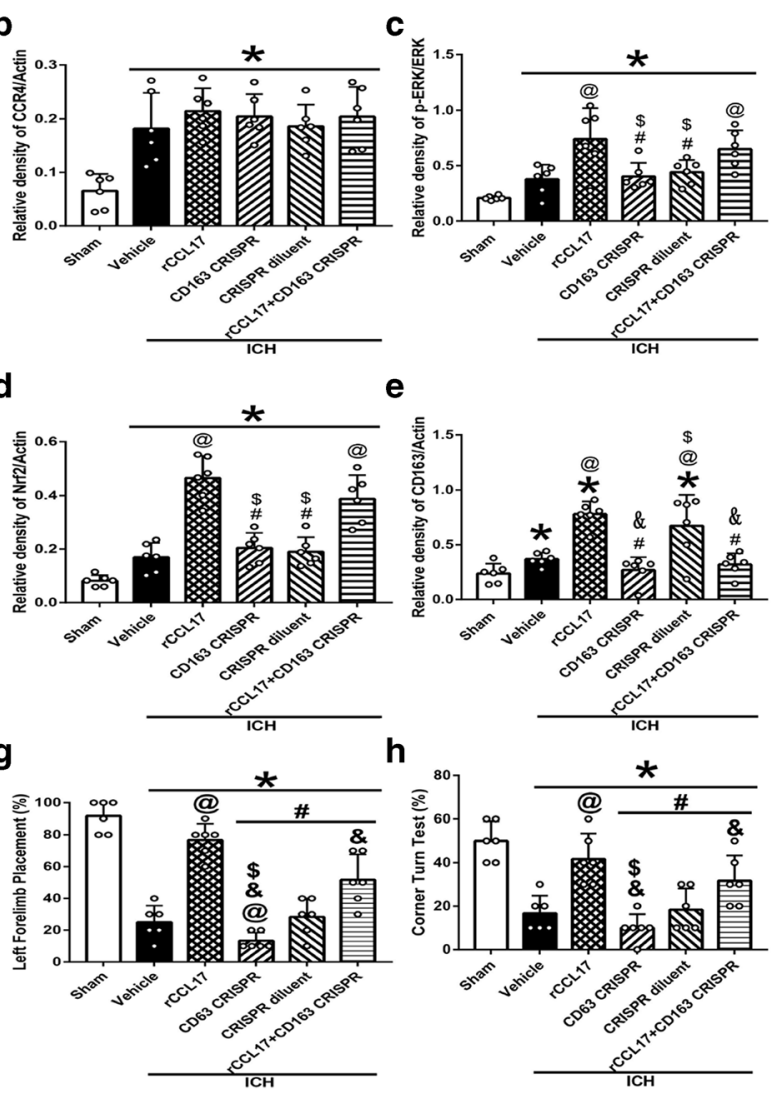

$\mathrm{ICH}+\mathrm{rCCL} 17, \$ p<0.05$ versus $\mathrm{ICH}+\mathrm{rCCL} 17+\mathrm{CD} 163$ CRISPR, $\& p<0.05$ versus $\mathrm{ICH}+\mathrm{CRISPR}$ diluent. Mean $\pm \mathrm{SD}$, one-way ANOVA, Tukey test, $n=6 /$ group. p-ERK1/2, phosphorylated extracellular regulated protein kinases $1 / 2$; ERK1/2, extracellular regulated protein kinases $1 / 2$

hematoma resolution after ICH which reduces neuronal necrosis caused by the direct compression effects of the hematoma and alleviates disruption of the BBB, thereby reducing brain edema post-ICH; (b) rCCL17 promoted hematoma resolution which reduces erythrocyte lysis products and consequently the secondary inflammatory response which leads to secondary brain injury after ICH is attenuated; (c) elevated CCL17 may promote chemotactic peripheral Treg cells into the brain, which inhibit the inflammatory response post-ICH and promoted polarized M1 microglia into M2 phenotype [47]. Modulation of the microglial phenotype might not only accelerate the absorption of hematoma but also improve white matter integrity, brain repair, and functional recovery which may contribute to improved long-term neurobehavioral outcomes after ICH.

The receptor CCR4 is a high-affinity receptor for CCL17 [48]. Previous studies showed that CCL17/CCR4 axis played an important role in tumor immunity and inflammatory diseases $[16,48]$. We observed that CCR4 expression increased after ICH. An increase in CCR4 expression may be necessary to carry out its function after the increase in expression of 
CCL17 after ICH. This may be a possible explanation for the increase in CCR4 expression after ICH. Nrf2 orchestrates a multifaceted response to oxidative stress in many diseases. Activated Nrf2 by cellular oxidative stress or electrophiles translocates to the nucleus and binds to antioxidant response elements which will upregulate HO-1 expression. HO-1 then is required for the increase in CD163 expression. Boyle et al. found the increase in CD163 after Nrf2 activation in the presence of excess heme may be via increasing expression of HO1 [49]. Previous publication showed that Nrf2, the downstream factor of ERK activation [50], promoted activated microglial clearing the hematoma by increasing CD163 expression on microglial/macrophages [51]. In our study, we did not detect the expression of HO-1 and oxidative stress factors in vivo, because we only focused on the hematoma clearance effects of activation of Nrf2 after $\mathrm{ICH}$, and we cannot exclude the possibility that activation of Nrf2 may have also exerted other protective effects.

CD163 was one of the markers expressed in M2-like microglia/macrophages [24] and played an important role in phagocytosis post-ICH. Our results showed that rCCL17 treatment increased the cellular expression of CD163 on activated microglia after ICH which was dependent on CCR4 mediated downstream activation of ERK1/2 and Nrf2. The increase in CD163-positive cells after rCCL17 treatment indicated a shift towards M2-like microglia/macrophages thereby promoting phagocytosis and hematoma clearance after ICH. Leclerc et al. reported a biphasic effect of CD163 receptor in collagenase induced ICH mouse model. CD163 deficiency had early beneficial properties at 3 days but delayed injurious effects at 10-day post-ICH [52]. We found that rCCL17 treatment increased the cellular expression of CD163 on activated microglia at $72 \mathrm{~h}$ after ICH, which contributed to hematoma clearance. ICH mice treated with rCCL17 had smaller hematoma volume and lower hemoglobin concentration observed at $72 \mathrm{~h}$ and 7 days after ICH. This difference in the role of CD163 maybe explained possibly due to different models used in our study and by Leclerc et al., autologous blood injection model may cause a mild secondary inflammatory response than collagenase model does. Second, the pathophysiology of neuroinflammation after ICH is a complex network, and CD163 KO may lead to a potential compensatory mechanism involved in anti-inflammation. In addition, significant proliferation of astrocytes after ICH observed in CD163 $\mathrm{KO}$ mice, which may promote the removal of hematoma and thus have a protective effect on inflammation.

Recent study by Liu et al. found CD163 was expressed in neurons and upregulated after ICH [53]. In the present study, we found some CD163+ cells are CD11b negative. We further performed additional immunofluorescence staining found CD163+ cells was co-localization with neurons (data did not show), which was consistent with Liu et al. But we focused on
CD163 expression on microglial/macrophages to promote hematoma resolution. Further studies need to be conducted to explore other potential effect of CD163 expression on neurons. Furthermore, we observed that the knockdown of CD163 reduced CD163 expression in brain tissue affected by ICH and eliminated the protective effects on short-term neurological function observed with rCCL17 treatment. Taken together, the current findings provide new sights on the role of rCCL17 in promoting hematoma clearance after ICH by stimulating CD163 expression through CCR4/ERK/ Nrf2 signaling pathway.

There are some limitations in the study. First, we did not monitor the expression of CCL17 in the serum and CSF after ICH. Second, we did not test each protein at the gene level. Third, it was reported that activation of Nrf2 upregulated CD36 expression on microglia, so we cannot exclude the possibility that rCCL17 enhances hematoma clearance through the Nrf2/CD36 pathway. In addition, the present study provides an association link between CCR4/ERK/Nrf2/CD163 signal activation in improving hematoma, and future studies should consider using different CCR4/ERK/Nrf2/CD163 signal pathway inhibitors to examine hematoma and functional outcomes in rCCL17-treated mice. Lastly, CCL17 may exert effects on T cells and the molecular mechanism of Treg cells regulating the phenotypic shift from M1-like microglia to M2like microglia was not evaluated and needs to be resolved in future studies.

\section{Conclusions}

We demonstrated that rCCL17 administration improved neurological function, reduced brain edema, and promoted hematoma clearance, which was associated with the activation of CCR4/ERK/Nrf2/CD163 signaling pathway after ICH. CCL17 may provide a novel therapeutic strategy against hematoma after ICH.

Authors' Contributions SXD and PS worked on the experimental design. SXD, PJ, and ZDT conducted the experiments. HL analyzed the data and drafted manuscript. JHZ, YG, and JPT participated in the experimental design and manuscript preparation.

Funding Information This study is supported partially by grants from the National Institutes of Health (NS082184 to John Zhang, NS101284 to Jiping Tang) and by grants from the National Key R\&D Program of China (2018YFC1312600, 2018YFC1312604 to Y. Gong).

\section{Compliance with Ethical Standards}

Competing Interests The authors declare that they have no competing interests.

Abbreviations $I C H$, intracerebral hemorrhage; $C C L 17$, C-C chemokine ligand17; $r C C L 17$, recombination $\mathrm{C}-\mathrm{C}$ chemokine ligand17; CCR4, C-C 
chemokine receptor 4; $N r f 2$, nuclear factor erythroid 2-related factor 2; IF, immunofluorescence; $H V$, hematoma volume; $H C$, hemoglobin content; $C R I S P R$, clustered regularly interspaced short palindromic repeats; DMSO, dimethyl sulfoxide; i.n., intranasally; i.c.v., intracerebroventricularly; $p$-ERKl/ 2 , phosphorylated extracellular regulated protein kinases $1 / 2 ; E R K 1 / 2$, extracellular regulated protein kinases $1 / 2 ; C N S$, central nervous system; $H b$, hemoglobin; $W W$, wet weight; $D W$, dry weight; $P B S$, phosphate-buffered saline; $B B B$, blood-brain barrier; $H O-1$, heme oxygenase-1

\section{References}

1. Qureshi AI, Tuhrim S, Broderick JP, Batjer HH, Hondo H, Hanley DF. Spontaneous intracerebral hemorrhage. The New England journal of medicine. May 10 2001;344(19):1450-1460.

2. Hemphill JC, 3rd, Greenberg SM, Anderson CS, et al. Guidelines for the Management of Spontaneous Intracerebral Hemorrhage: A Guideline for Healthcare Professionals From the American Heart Association/American Stroke Association. Stroke. Jul 2015;46(7): 2032-2060.

3. Zhang $\mathrm{S}, \mathrm{Hu} \mathrm{ZW}$, Luo HY, et al. AAV/BBB-Mediated Gene Transfer of CHIP Attenuates Brain Injury Following Experimental Intracerebral Hemorrhage. Translational stroke research. Jul 192019.

4. Wang G, Guo Z, Tong L, et al. TLR7 (Toll-Like Receptor 7) Facilitates Heme Scavenging Through the BTK (Bruton Tyrosine Kinase)-CRT (Calreticulin)-LRP1 (Low-Density Lipoprotein Receptor-Related Protein-1)-Hx (Hemopexin) Pathway in Murine Intracerebral Hemorrhage. Stroke. Dec 2018;49(12):3020-3029.

5. Tao C, Keep RF, Xi G, Hua Y. CD47 Blocking Antibody Accelerates Hematoma Clearance After Intracerebral Hemorrhage in Aged Rats. Translational stroke research. Oct 302019.

6. Zhao X, Sun G, Zhang J, et al. Hematoma resolution as a target for intracerebral hemorrhage treatment: role for peroxisome proliferator-activated receptor gamma in microglia/macrophages. Annals of neurology. Apr 2007;61(4):352-362.

7. Adeoye O, Broderick JP. Advances in the management of intracerebral hemorrhage. Nature reviews. Neurology. Nov 2010;6(11): 593-601.

8. Wu X, Luo J, Liu H, et al. Recombinant Adiponectin Peptide Ameliorates Brain Injury Following Intracerebral Hemorrhage by Suppressing Astrocyte-Derived Inflammation via the Inhibition of Drp1-Mediated Mitochondrial Fission. Translational stroke research. Jan 42020.

9. Mendelow AD, Gregson BA, Rowan EN, Murray GD, Gholkar A, Mitchell PM. Early surgery versus initial conservative treatment in patients with spontaneous supratentorial lobar intracerebral haematomas (STICH II): a randomised trial. Lancet (London, England). Aug 3 2013;382(9890):397-408.

10. Gonzales NR. Ongoing clinical trials in intracerebral hemorrhage. Stroke. Jun 2013;44(6 Suppl 1):S70-73.

11. Tan Q, Guo P, Zhou J, et al. Targeting neutrophil extracellular traps enhanced tPA fibrinolysis for experimental intracerebral hemorrhage. Translational research : the journal of laboratory and clinical medicine. Sep 2019;211:139-146.

12. Zhang Z, Cho S, Rehni AK, Quero HN, Dave KR, Zhao W. Automated Assessment of Hematoma Volume of Rodents Subjected to Experimental Intracerebral Hemorrhagic Stroke by Bayes Segmentation Approach. Translational stroke research. Dec 142019.

13. Griffith JW, Sokol CL, Luster AD. Chemokines and chemokine receptors: positioning cells for host defense and immunity. Annual review of immunology. 2014;32:659-702.
14. Flynn G, Maru S, Loughlin J, Romero IA, Male D. Regulation of chemokine receptor expression in human microglia and astrocytes. Journal of neuroimmunology. Mar 2003;136(1-2):84-93.

15. Ishida T, Ueda R. CCR4 as a novel molecular target for immunotherapy of cancer. Cancer science. Nov 2006;97(11):1139-1146.

16. Scheu S, Ali S, Ruland C, Arolt V, Alferink J. The C-C Chemokines CCL17 and CCL22 and Their Receptor CCR4 in CNS Autoimmunity. International journal of molecular sciences. Nov 2 2017;18(11).

17. Fulle L, Offermann N, Hansen JN, et al. CCL17 exerts a neuroimmune modulatory function and is expressed in hippocampal neurons. Glia. Oct 2018;66(10):2246-2261.

18. Yoshie O, Imai T, Nomiyama H. Novel lymphocyte-specific CC chemokines and their receptors. Journal of leukocyte biology. Nov 1997;62(5):634-644.

19. Cheng X, Wu H, Jin ZJ, et al. Up-regulation of chemokine receptor CCR4 is associated with Human Hepatocellular Carcinoma malignant behavior. Scientific reports. Sep 28 2017;7(1):12362.

20. Cen J, Zhao N, Huang WW, et al. Polyamine analogue QMA attenuated ischemic injury in MCAO rats via ERK and Akt activated Nrf2/HO-1 signaling pathway. European journal of pharmacology. Feb 5 2019;844:165-174.

21. Flores JJ, Klebe D, Tang J, Zhang JH. A comprehensive review of therapeutic targets that induce microglia/macrophage-mediated hematoma resolution after germinal matrix hemorrhage. Journal of neuroscience research. Jan 2020;98(1):121-128.

22. Kristiansen $\mathrm{M}$, Graversen $\mathrm{JH}$, Jacobsen $\mathrm{C}$, et al. Identification of the haemoglobin scavenger receptor. Nature. Jan 11 2001;409(6817): 198-201.

23. Cao S, Zheng M, Hua Y, Chen G, Keep RF, Xi G. Hematoma Changes During Clot Resolution After Experimental Intracerebral Hemorrhage. Stroke. Jun 2016;47(6):1626-1631.

24. Garton T, Keep RF, Hua Y, Xi G. CD163, a Hemoglobin/ Haptoglobin Scavenger Receptor, After Intracerebral Hemorrhage: Functions in Microglia/Macrophages Versus Neurons. Translational stroke research. Dec 2017;8(6):612-616.

25. Yan J, Zuo G, Sherchan P, et al. CCR1 Activation Promotes Neuroinflammation Through CCR1/TPR1/ERK1/2 Signaling Pathway After Intracerebral Hemorrhage in Mice. Neurotherapeutics : the journal of the American Society for Experimental NeuroTherapeutics. Jan 2020.

26. Liu LB, Xie F, Chang KK, et al. Chemokine CCL17 induced by hypoxia promotes the proliferation of cervical cancer cell. American journal of cancer research. 2015;5(10):3072-3084.

27. McMillin M, Frampton G, Thompson M, et al. Neuronal CCL2 is upregulated during hepatic encephalopathy and contributes to microglia activation and neurological decline. Journal of neuroinflammation. Jul 10 2014;11:121.

28. Xian P, Hei Y, Wang R, et al. Mesenchymal stem cell-derived exosomes as a nanotherapeutic agent for amelioration of inflammation-induced astrocyte alterations in mice. Theranostics. 2019;9(20):5956-5975.

29. Manaenko A, Yang P, Nowrangi D, et al. Inhibition of stress fiber formation preserves blood-brain barrier after intracerebral hemorrhage in mice. Journal of cerebral blood flow and metabolism : official journal of the International Society of Cerebral Blood Flow and Metabolism. Jan 2018;38(1):87-102.

30. Li P, Zhao G, Ding Y, et al. Rh-IFN-alpha attenuates neuroinflammation and improves neurological function by inhibiting NF-kappaB through JAK1-STAT1/TRAF3 pathway in an experimental GMH rat model. Brain, behavior, and immunity. Jul 2019;79:174-185.

31. Krafft PR, Caner B, Klebe D, Rolland WB, Tang J, Zhang JH. PHA-543613 preserves blood-brain barrier integrity after intracerebral hemorrhage in mice. Stroke. Jun 2013;44(6):1743-1747.

32. Lekic T, Manaenko A, Rolland W, et al. Rodent neonatal germinal matrix hemorrhage mimics the human brain injury, neurological 
consequences, and post-hemorrhagic hydrocephalus. Experimental neurology. Jul 2012;236(1):69-78.

33. Flores JJ, Klebe D, Rolland WB, Lekic T, Krafft PR, Zhang JH. PPARgamma-induced upregulation of CD36 enhances hematoma resolution and attenuates long-term neurological deficits after germinal matrix hemorrhage in neonatal rats. Neurobiology of disease. Mar 2016;87:124-133.

34. Mo J, Enkhjargal B, Travis ZD, et al. AVE 0991 attenuates oxidative stress and neuronal apoptosis via Mas/PKA/CREB/UCP-2 pathway after subarachnoid hemorrhage in rats. Redox biology. Jan 2019;20:75-86.

35. Peng J, Pang J, Huang L, et al. LRP1 activation attenuates white matter injury by modulating microglial polarization through Shc1/PI3K/Akt pathway after subarachnoid hemorrhage in rats. Redox biology. Feb 2019;21:101121.

36. Zhu H, Wang Z, Yu J, et al. Role and mechanisms of cytokines in the secondary brain injury after intracerebral hemorrhage. Progress in neurobiology. Jul 2019;178:101610.

37. Guo G, Pan C, Guo W, et al. Efficacy and safety of four interventions for spontaneous supratentorial intracerebral hemorrhage: a network meta-analysis. Journal of neurointerventional surgery. Jan 32020.

38. Hostettler IC, Morton MJ, Ambler G, et al. Haptoglobin genotype and outcome after spontaneous intracerebral haemorrhage. Journal of neurology, neurosurgery, and psychiatry. Jan 102020.

39. Lan X, Han X, Li Q, Yang QW, Wang J. Modulators of microglial activation and polarization after intracerebral haemorrhage. Nature reviews. Neurology. Jul 2017;13(7):420-433.

40. Michell-Robinson MA, Touil H, Healy LM, et al. Roles of microglia in brain development, tissue maintenance and repair. Brain : a journal of neurology. May 2015;138(Pt 5):1138-1159.

41. Chang CF, Wan J, Li Q, Renfroe SC, Heller NM, Wang J. Alternative activation-skewed microglia/macrophages promote hematoma resolution in experimental intracerebral hemorrhage. Neurobiology of disease. Jul 2017;103:54-69.

42. Kim E, Cho S. Microglia and Monocyte-Derived Macrophages in Stroke. Neurotherapeutics : the journal of the American Society for Experimental NeuroTherapeutics. Oct 2016;13(4):702-718.

43. Murray PJ, Wynn TA. Protective and pathogenic functions of macrophage subsets. Nature reviews. Immunology. Oct 14 2011;11(11): 723-737.
44. Hammond MD, Ai Y, Sansing LH. Gr1+ Macrophages and Dendritic Cells Dominate the Inflammatory Infiltrate 12 Hours After Experimental Intracerebral Hemorrhage. Translational stroke research. Jul 2012;3(1):s125-s131.

45. Gong C, Hoff JT, Keep RF. Acute inflammatory reaction following experimental intracerebral hemorrhage in rat. Brain research. Jul 14 2000;871(1):57-65.

46. Sakane T, Akizuki M, Yamashita S, Nadai T, Hashida M, Sezaki H. The transport of a drug to the cerebrospinal fluid directly from the nasal cavity: the relation to the lipophilicity of the drug. Chemical \& pharmaceutical bulletin. Sep 1991;39(9):2456-2458.

47. Mao LL, Yuan H, Wang WW, et al. Adoptive Regulatory T-cell Therapy Attenuates Perihematomal Inflammation in a Mouse Model of Experimental Intracerebral Hemorrhage. Cellular and molecular neurobiology. Jul 2017;37(5):919-929.

48. Yoshie O, Matsushima K. CCR4 and its ligands: from bench to bedside. International immunology. Jan 2015;27(1):11-20.

49. Boyle JJ, Johns M, Lo J, et al. Heme induces heme oxygenase 1 via Nrf2: role in the homeostatic macrophage response to intraplaque hemorrhage. Arteriosclerosis, thrombosis, and vascular biology. Nov 2011;31(11):2685-2691.

50. Zhao H, Garton T, Keep RF, Hua Y, Xi G. Microglia/Macrophage Polarization After Experimental Intracerebral Hemorrhage. Translational stroke research. Dec 2015;6(6):407-409.

51. Bulters D, Gaastra B, Zolnourian A, et al. Haemoglobin scavenging in intracranial bleeding: biology and clinical implications. Nature reviews. Neurology. Jul 2018;14(7):416-432.

52. Leclerc JL, Lampert AS, Loyola Amador C, et al. The absence of the CD163 receptor has distinct temporal influences on intracerebral hemorrhage outcomes. Journal of cerebral blood flow and metabolism : official journal of the International Society of Cerebral Blood Flow and Metabolism. Feb 2018;38(2):262-273.

53. Liu R, Cao S, Hua Y, Keep RF, Huang Y, Xi G. CD163 Expression in Neurons After Experimental Intracerebral Hemorrhage. Stroke. May 2017;48(5):1369-1375.

Publisher's Note Springer Nature remains neutral with regard to jurisdictional claims in published maps and institutional affiliations. 